\title{
NOMMER LES VIOLENCES SCOLAIRES. DES INCIVILITÉS AU HARCĖLEMENT SCOLAIRE
}

\author{
$\underline{\text { Claire de Saint Martin }}$
}

Érès | La lettre de l'enfance et de l'adolescence »

2012/1 n $87 \mid$ pages 119 à 126

ISSN 2101-6046

ISBN 9782749216430

Article disponible en ligne à l'adresse :

https://www.cairn.info/revue-lettre-de-l-enfance-et-de-ladolescence-2012-1-page-119.htm

Distribution électronique Cairn.info pour Érès.

(C) Érès. Tous droits réservés pour tous pays.

La reproduction ou représentation de cet article, notamment par photocopie, n'est autorisée que dans les limites des conditions générales d'utilisation du site ou, le cas échéant, des conditions générales de la licence souscrite par votre établissement. Toute autre reproduction ou représentation, en tout ou partie, sous quelque forme et de quelque manière que ce soit, est interdite sauf accord préalable et écrit de l'éditeur, en dehors des cas prévus par la législation en vigueur en France. Il est précisé que son stockage dans une base de données est également interdit. 


\section{Nommer les violences scolaires Des incivilités au harcèlement scolaire}

\section{Claire de Saint Martin}

Le thème de la violence scolaire fait florès dans les médias depuis les années 1990, repris par un certain nombre de chercheurs, dont l'un des plus emblématiques est sans nul doute Éric Debarbieux, président de l'Observatoire international de la violence à l'école, qui a présidé les dernières Assises nationales sur le harcèlement scolaire les 2 et 3 mai 2011. Cette nouvelle dénomination interroge sur l'évolution de la réflexion sur les violences scolaires. En effet, la caractérisation de celles-ci a évolué depuis la prise en compte de cette question dans les débats publics. Si les dénominations suivantes ont coexisté, la prédominance de l'une ou de l'autre renseigne sur les représentations sociales de la violence. Du terme d' «incivilités », apparu dans les années 1990, on est passé à celui de "microviolences » dans les années 2000. Aujourd'hui, les recherches se recentrent sur le harcèlement scolaire. Que signifie ce changement de nom et qu'annonce-t-il en termes de traitement de la violence à l'école?

\section{Les incivilités}

La notion d' "incivilités » a vu le jour en mars 1982 avec la parution de l'article de Wilson \& Ketting "Broken Windows ", dans lequel les auteurs exposent une théorie sur le désordre : si on laisse dans un quartier une vitre brisée, toutes les autres connaîtront le même sort. De même, laisser impunis les petits délits quotidiens amène forcément leurs auteurs à commettre des infractions de plus en plus graves. Il s'agit donc d'endiguer les infractions graves en punissant les premiers délits. Roché, le premier à avoir utilisé ce terme en France, le définit par des atteintes à l'ordre public du quotidien ordinaire. Il en distingue quatre catégories : dégradation et vandalisme,

Claire de Saint Martin, doctorante en sciences de l'éducation, laboratoire EMA, université de CergyPontoise. 
manque de courtoisie et insultes, conflits autour du bruit, comportements perturbateurs et occupation de l'espace (Roché, 1993).

$\mathrm{Au}$ sein de l'école, le terme désigne donc tous les faits, les désordres, souvent impunis du fait de leur invisibilité par les adultes ou de leur caractère jugé mineur, qui contribuent à alourdir l'atmosphère scolaire, à nuire au déroulement fluide de la classe : chahuts, impolitesses, humiliations, paroles blessantes... Ces faits, sans relever du pénal, constituent des atteintes à la personne et à la collectivité.

Même mineures, les conséquences de ces incivilités, de par leur réitération quotidienne, peuvent engendrer des conséquences individuelles et collectives non négligeables : sentiment d'insécurité, d'abandon, d'impuissance et repli sur soi pour les victimes, dégradation du climat scolaire, désertion des espaces publics par les enseignants et augmentation de la violence pour le collectif (Blaya, 2006 ; Debarbieux, Dupuch, Montoya, 1997 ; Debarbieux, 2006). «L'incivilité est la révélation d'un chaos possible, une perte de sens et de confiance en soi et dans les autres. Bref, entre le tout du crime et le néant du fantasme, il y a une place pour une insécurité au quotidien ${ }^{1}$.»

Le mot affirme l'idée d'une violence scolaire qui ne se réduit pas aux crimes et aux délits, et désigne tous les faits délictueux qui ont lieu au sein de l'école, de l'insolence aux agressions.

Il marque la volonté de traiter tous les types de violence existants ou perçus, et en élargit l'empan hors du champ pénal. On voit alors comment la question peut devenir centrale dans le domaine scolaire.

Le terme est cependant doublement critiqué, en raison à la fois de la survalorisation des faits désignés et de leur sous-valorisation. En effet, il peut mésestimer la gravité des faits, par leur minimisation et leur banalisation. Le terme ne couvre pas les mêmes représentations en France et aux États-Unis, pays où les incivilités sont plus répréhensibles et sévèrement réprimées. Â l'opposé, l'emploi du terme peut conduire à une criminalisation des comportements ordinaires (Wacquant, 1999) et induit un caractère délinquant excessif au regard des faits observés. Éric Debarbieux, après l'avoir revendiquée, en dénonce l'utilisation : «L'usage excessif de la notion d'incivilité entraîne une surqualification des désordres ou des indisciplines, tout en s'accentuant vers un culturalisme xénophobe ${ }^{2}$. » Philippe Vienne précise : "Les incivilités constituent donc une catégorie tellement ouverte et étendue qu'elle perd automatiquement toute pertinence ${ }^{3}$.»

La métaphore de la « vitre brisée » montre aussi ses limites : l'incivilité ne mène pas inéluctablement à la délinquance, et la confusion possible entre acte et identité a conduit les experts à renoncer à cette désignation des violences scolaires.

1. E. Debarbieux, A. Dupuch, Y. Montoya, « Pour en finir avec le "handicap socioviolent" : une approche comparative de la violence en milieu scolaire », dans B. Charlot, J.-C. Emin (sous la direction de), Violences à l'école, État des savoirs, Paris, Armand Colin, 1997, p. 19.

2. E. Debarbieux, Violence à l'école : un défi mondial ?, Paris, Armand Colin, 2006, p. 114.

3. P. Vienne, Violences à l'école : au bonheur des experts, Paris, Syllepse, 2009, p. 140. 


\section{Les microviolences}

Éric Debarbieux a alors proposé le terme de microviolence pour désigner les désordres et infractions quotidiennes de l'école. Il s'agissait de décrire toutes les violences mineures qui empoisonnent au quotidien la vie scolaire et contribuent à en dégrader le climat. Cette notion fait « référence à la fois aux péri-délits et aux microvictimations, aux délits sans victimes directes, à la répétitivité et la fréquence de désordres et infractions parfois ténus et inaperçus qui, lorsqu'ils ne sont pas pris en compte, perturbent l'ordre scolaire et rendent la vie en collectivité difficile, voire impossible ${ }^{4}$ ». Ces microviolences concernent toutes les interactions à l'intérieur de l'école : entre élèves et professeurs (injures, humiliations, refus d'écouter et/ ou de travailler, indiscipline), entre pairs (bousculades, intimidations, harcèlements). Elles peuvent être individuelles ou collectives, adressées ou non (chahut) et se caractérisent par leur répétitivité. Elles sont principalement responsables de l'expérience victimaire précoce (Debarbieux, 2006).

On retrouve ici les catégories délictueuses définies dans le terme " incivilités ». Si la désignation change, le contenu reste identique. Le terme « microviolences » peut donc recevoir la même critique de Philippe Vienne, du fait des mêmes imprécisions, de l'absence de hiérarchisation de ces délits : le bruit, majoritairement dénoncé comme source première de violence par les enseignants (Debarbieux, 1990), relève-il d'un acte délibérément nocif des élèves ? Peut-on accorder la même valeur à un coup porté intentionnellement et à une bousculade involontaire?

Le changement de caractérisation des violences scolaires accompagne la dramatisation médiatique, tout en s'en défendant : le mot « violence » est explicitement revendiqué par les experts ; le préfixe «micro » inscrit les faits dans le quotidien ordinaire de l'école, mais minimise aussi la charge émotionnelle de substantif, dénonçant ainsi les dangers d'une dramatisation par la médiatisation et les récupérations politiques du thème (Blaya, 2006 ; Carra, 2009 ; Debarbieux, 1990, 1997, 2006).

Le terme, recevant les mêmes critiques que celui d'incivilités, semble aujourd'hui connaître le même sort : dans le dernier dossier paru sur le sujet, seul Éric Debarbieux l'emploie pour désigner les violences scolaires ${ }^{5}$. Il est également significatif que les Assises nationales sur le harcèlement scolaire, qui se sont tenues les 2 et 3 mai 2011 sous la tutelle du ministère de l'Éducation nationale, aient supprimé le mot « microviolences » des débats. Cette disparition et le nouveau titre donné à ces Assises semblent opérer une évolution significative du traitement des violences scolaires : le harcèlement scolaire est la traduction du terme anglais school bullying, qui en décrit une forme particulière.

4. C. Blaya, Violences et maltraitances en milieu scolaire, Paris, Armand Colin, 2006, p. 21.

5. La nouvelle revue de l'adaptation et de la scolarisation, $\mathrm{n}^{\circ} 53$, Les violences à l'école, Suresnes, Éditions de l'INS-HEA, mai 2011, p. 5 à 194. 


\section{Le school bullying}

Le concept de bullying date des années 1970 et a été systématiquement étudié par le Norvégien Dan Olweus, qui le définit comme une action négative portée par un ou plusieurs élèves à l'encontre d'un autre (Olweus, 1993). Ces actes sont peu visibles par les adultes et ont des conséquences multiples, qui vont du décrochage scolaire au suicide. Il concerne exclusivement les relations entre pairs, dans un rapport de force inégal où l'agresseur abuse de sa domination sur la victime. Il n'y a pas de bullying entre deux élèves de force égale. La volonté de nuire est à l'origine de ces actes, qui se caractérisent par leur répétitivité et leur durée sur le long terme. Il s'agit donc d'un abus de pouvoir réfléchi et structuré qui vise à la destruction de la victime. Ses multiples manifestations peuvent être physiques (coups, bousculades, coups de pied, pincements, maitrise physique de l'autre), verbales (menaces, railleries, moqueries, surnoms dépréciatifs), mais relèvent aussi du non-verbal : regards insistants, grimaces, gestes menaçants ${ }^{6}$. L'école primaire et le collège sont les lieux privilégiés du bullying qui décroît avec l'âge.

D'après Olweus, le bullying est d'abord le fait des garçons, mais les filles peuvent aussi l'exercer. Les agresseurs sont souvent plus âgés que leurs victimes. Il en distingue deux types : le bullying direct et le bullying indirect. Le premier désigne les actes physiques, les attaques directes sur les victimes. Les garçons en sont majoritairement coupables et victimes. Le bullying indirect est une forme plus subtile de violence : il s'agit ici d'isoler la victime du groupe par le harcèlement, la diffamation, les rumeurs, la manipulation des autres contre la victime. Selon lui, les filles utilisent plus ce bullying, mais les garçons en sont tout autant victimes qu'elles. Le bullying peut être réactif à une frustration ou une agression, ou proactif dans le but d'obtenir quelque chose, un bien matériel ou une reconnaissance sociale.

Olweus établit un profil précis de l'agresseur et de la victime. Les agresseurs ont souvent des relations conflictuelles avec leurs parents et les adultes en général. Ils ont une vision positive de la violence et sont souvent caractérisés par leur impulsivité et le besoin de dominer autrui. Ils éprouvent peu d'empathie pour les victimes, ne connaissent pas l'anxiété et ne souffrent pas d'une mésestime de soi. Ils sont physiquement plus forts que leurs victimes, surtout les garçons. Les victimes sont toujours plus faibles que leur(s) agresseur(s). Elles manquent de confiance, ont une mauvaise image d'ellesmêmes et sont angoissées. Elles ont du mal à trouver leur place dans un groupe et sont souvent isolées, sont sans défense face à leur agresseur. Leurs relations avec leurs parents sont bonnes, surtout avec leur mère.

Ce concept rencontre un grand succès dans les pays nordiques et anglosaxons, aux Pays-Bas, aux États-Unis et au Canada, ainsi qu'en Asie (Corée du Sud, Japon). Il peine à être considéré en France, malgré les efforts de 
Jacques Pain qui a fait traduire le livre d'Olweus. C'est pourquoi aucune traduction française du terme ne semble satisfaisante. Le Québec le traduit par « intimidation », mais ce terme réduit le fait à une dimension qui relève plus de la menace que de l'acte. La Belgique francophone utilise le mot "brimade ", mettant ainsi l'accent sur l'humiliation et le déséquilibre des forces entre les protagonistes, en oubliant les autres facteurs du bullying. La traduction communément employée est donc celle du titre du livre d'Olweus, proposée par Jacques Pain : Violences entre élèves, harcèlements et brutalités entre élèves (1999).

Le concept de bullying réintroduit l'intentionnalité de l'acte malveillant dans la définition des violences scolaires. Mais les violences entre pairs ne se caractérisent pas toutes par un déséquilibre des forces et ne se définissent pas uniquement dans un rapport exclusif agresseur/victime (Carra, Sicot, 1997). Dans la cour de récréation, un élève vient en pleurant dénoncer un pair qui l'a tapé. Les larmes accordent rapidement le statut de victime à celui qui les verse. Mais l'élève incriminé justifie son acte par une insulte proférée à son encontre par la victime. La violence exercée ici se joue dans une réciprocité, où la victime est aussi l'agresseur.

De plus, la caractérisation des protagonistes conduit à leur psychologisation, par l'identification d'une responsabilité individuelle trop réductrice. Cette psychologisation comporte aussi une dimension prédictive : Olweus lui-même signale que les agresseurs ont tendance à tomber dans la délinquance. Le risque identitaire du phénomène devient alors patent. La responsabilité des violences scolaires incombe désormais à des individus désignés et reconnus.

Le harcèlement scolaire désigne exclusivement les violences entre élèves, peu abordé dans les recherches scientifiques. De ce point de vue, il témoigne de la volonté de prise en charge de types de violences jusque-là peu étudiées en France. Mais en même temps, il réduit ces violences aux seuls faits entre pairs, dans un temps restreint, puisque le school bullying ne concerne que les élèves du primaire et du secondaire. Il évacue donc tout autre type de violence, et notamment la violence institutionnelle.

\section{Une responsabilité individuelle}

Depuis la médiatisation du phénomène des violences scolaires, aucune définition donnée ne semble donc satisfaisante. Les termes d'incivilités et de microviolences sont caducs, celui de harcèlement scolaire est trop réducteur pour rendre compte de la nature exacte de ces violences. En même temps, que désigne l'évolution de ces dénominations en terme d'enjeux et de représentations sociales?

Ces caractérisations font porter de plus en plus précisément la responsabilité des violences scolaires sur l'individu. Leur évolution révèle celle des volontés politiques. En novembre 2010, le rapport de Jean-Marie Bockel au président de la République, La prévention de la délinquance des jeunes, relève que « les études démontrent en effet que $15 \%$ des enfants sont vulnérables, 
c'est-à-dire incapables de répondre, de manière adaptée, à l'agressivité interne ou externe à laquelle ils sont exposés [...] Cette vulnérabilité pourrait pourtant être repérée chez les petits entre deux et trois ans. C'est à ce stade que doit être posé sur l'enfant un regard pluridisciplinaire visant à rechercher s'il existe à ces troubles une cause médicale ou familiale ${ }^{7}$ ». La nouvelle dénomination de harcèlement scolaire s'inscrit dans cette continuité, en focalisant le regard sur des comportements individuels, liés à un type de personnalité. Le fait que le harcèlement scolaire ne concerne que les violences entre pairs accentue l'idée d'une violence exclusivement due aux élèves.

Si tous les chercheurs dénoncent les dangers de la dramatisation médiatique, certains d'entre eux accompagnent le sentiment d'insécurité collectif et y participent. Ne traiter que le harcèlement scolaire stigmatise des individus en devenir et évacue la question principale : la violence est un fait social (Wieviorka, 2005 ; Monceau, 2011). En effet « la violence n'est pas un concept, mais un objet social, un sujet de préoccupation ${ }^{8} »$. Identifier les violences scolaires au seul comportement des élèves en ignore la dimension interrelationnelle et sociale, parce que cela décontextualise les faits, pour se centrer sur un seul type de comportement.

\section{Les enquêtes de victimation}

Cette orientation de la recherche est cependant en accord avec la méthodologie prônée par Éric Debarbieux et ratifiée par le ministère de l'Éducation nationale pour étudier ces violences : les enquêtes de victimation. « L'enquête de victimation consiste à appréhender les transgressions et les infractions, non plus du point de vue des agences de contrôle social mais du point de vue de l'un des acteurs principaux, un informateur privilégié, direct : la victime ${ }^{9}$. » Les enquêtes de victimation sont nées aux États-Unis dans les années 1960, à la demande du président Johnson, pour mieux connaître la criminalité (Wieviorka, 2005). Elles sont utilisées dans le domaine scolaire en France depuis les années 1990. La première a été réalisée par Cécile Carra et François Sicot dans le Doubs en 1997, et concernait les établissements du secondaire. Elles doivent permettre de rendre compte de façon plus exhaustive du phénomène de la violence à l'école, en considérant son impact physique et psychologique sur les victimes. Elles participent d'une vision subjective des violences, en ne tenant compte que de la perception qu'en ont les victimes. Éric Debarbieux appelle violence « ce qui m'arrive et que je perçois comme telle ${ }^{10}$ ». Catherine Blaya et Cécile Carra

\footnotetext{
7. J.-M. Bockel, secrétaire d'État à la Justice, La prévention de la délinquance des jeunes, Rapport à monsieur le président de la République, novembre 2010.

8. L. Catini, G. Monceau, «Socianalyse de la violence en éducation », La lettre du GRAPE, n 39, Traiter la violence, Toulouse, érès, 2000, p. 54.

9. C. Carra, F. Sicot, «Une autre perspective sur les violences scolaires : l'expérience de victimation », dans B. Charlot, J.-C. Emin (sous la direction de), Violences à l'écoles élémentaire, op. cit., p. 64.

10. E. Debarbieux, La violence dans la classe, Issy-les-Moulineaux, ESF, 1999, p. 19.
} 
affirment également la nécessité de considérer la violence perçue (Blaya, 2006 ; Carra, 2009).

Mais l'examen des violences à la seule lumière des "victimes " que proposent les enquêtes de victimation nous semble non seulement parcellaire mais également dangereux, parce qu'il en minore la dimension interrelationnelle et donne une prétention scientifique à la seule perception individuelle. Philippe Vienne écrit : «La violence est tout d'abord posée comme un donné, en vue de quantification. Mais ce qui sera éventuellement quantifié n'est ni nécessairement considéré comme "violence" par la population étudiée - ce que devrait montrer un abord compréhensif, s'il est réellement souhaité par l'auteur - ni nécessairement quantifiable, comme ce domaine des perceptions de la violence, sur lequel Debarbieux voudrait déceler une intensité mesurable ${ }^{11}$.»

\section{La responsabilité institutionnelle}

Les différentes dénominations étudiées tendent à évacuer toute responsabilité institutionnelle du phénomène, pour se centrer sur l'étude exclusive de faits et de comportements, jusqu'à ne considérer que les seuls actes des élèves par l'usage du terme de harcèlement scolaire.

Or, les violences scolaires s'inscrivent dans une institution définie, oubliée par la désignation de harcèlement scolaire. Elles sont, comme nous l'avons dit, une production sociale, en tant qu'expressions des contradictions et des dysfonctionnements de l'institution, une réponse à des situations données dans un cadre particulier. Nous rejoignons l'analyse de Laura Catini et Gilles Monceau : la question de la violence des élèves conduit ici à " des problèmes de communication et de relations interindividuelles, puis désaccords d'organisation de l'établissement et la perception de ses missions ${ }^{12} »$. De ce fait, on peut les considérer comme des analyseurs de l'institution elle-même, l'institution se définissant comme une dynamique où agissent tous les acteurs qui s'y trouvent, et où donc s'articulent des relations de pouvoir, des enjeux personnels et collectifs, affectifs, des contradictions multiples. C'est pourquoi il convient d'analyser plus précisément les différentes formes de violences scolaires, non seulement à l'aune de leur gravité et des individus, mais aussi en regard des circonstances de leur expression et de leurs enjeux.

Envisager les violences scolaires du seul point de vue des victimes et en stigmatisant un type d'élèves méconnaît l'importance de l'institution dans leurs manifestations, intègre un cadre normatif de plus en plus sévère, exigeant une conformité absolue des élèves à la " norme ", psychologise un fait social. C'est aussi aboutir à l'effet médiatique dénoncé par ces mêmes experts, la dramatisation des violences scolaires. « La violence est un concept mou au plan théorique, chargé au plan affectif et instrumentalisé au plan 
idéologique ${ }^{13}$. » Affirmer la nécessité de prévenir les violences scolaires sous peine de voir les enfants devenir des criminels, aux seules lumières du harcèlement scolaire et des enquêtes de victimation, c'est à coup sûr entrer dans le champ de l'instrumentalisation de la violence. Si les violences scolaires doivent faire l'objet d'un traitement éducatif et social, concernant tous les acteurs de l'institution, il est nécessaire de les envisager dans leur globalité (acteurs, victimes, contexte), et non du seul point de vue des victimes. Questionner les violences scolaires oblige donc d'abord à questionner l'institution, avant les individus.

\section{Bibliographie}

Blaya, C. 2006. Violences et maltraitances en milieu scolaire, Paris, Armand Colin.

Bockel, J.-M. 2010. La prévention de la délinquance des jeunes, Rapport à monsieur le président de la République.

Carra, C. 2009. Violences à l'école élémentaire, Paris, Puf.

CARra, C. ; Sicot, F. 1997. « Une autre perspective sur les violences scolaires : l'expérience de victimation », dans B. Charlot, J.-C. Emin (sous la direction de), Violences à l'école. État des savoirs, Paris, Armand Colin.

Catini, L. ; Monceau, G. 2000. "Socianalyse de la violence en éducation », Traiter la violence, La lettre du GRAPE, $\mathrm{n}^{\circ} 39$, Toulouse, érès.

Debarbieux, E. 1990. La violence dans la classe, Issy-les-Moulineaux, esf, 1999.

Debarbieux, E. 2006. Violence à l'école : un défi mondial ?, Paris, Armand Colin.

Debarbieux, E. ; Dupuch, A. ; Montoya, Y. 1997, « Pour en finir avec le "handicap socioviolent" : une approche comparative de la violence en milieu scolaire », dans B. Charlot, J.-C. Emin (sous la direction de), Violences à l'école. État des savoirs, Paris, Armand Colin.

La nouvelle revue de l'adaptation et de la scolarisation. Les violences à l'école, $\mathrm{n}^{\circ} 53$, Suresnes, Éditions de l'INS-HEA, mai 2011, p. 5 à 194.

Monceau, G. 2011. «Violence, urgence, efficience, excellence », Cahiers pédagogiques, n 488, Paris, CRAP-Cahiers pédagogiques.

Olweus, D. 1993. Bullying at School: What We Know, What We Can Do, Oxford (uk)-Cambridge (USA), Blackwell Publishers.

Rоввеs, B. 2010. L'autorité éducative dans la classe, Issy-les-Moulineaux, Esf éditeur.

Robbes, B. ; Bride, P. 2011. «Quelle violence? Quelle école? », Cahiers pédagogiques, nº 488, Paris, CRAP-Cahiers pédagogiques.

Roché, S. 1993. Le sentiment d'insécurité, Paris, puf.

Vienne, P. 2009. Violences à l'école : au bonheur des experts, Paris, Syllepse.

WaCQuant, L. 1999. Les prisons de la misère, Paris, Éditions Raisons d'agir.

Wieviorka, M. 2005. La violence, Paris, Hachette Littératures, coll. «Pluriel ».

Wilson, J.Q. ; Kelling, G. 1982. «Broken Windows », Atlantic Monthly, mars, p. 29-38.

13. B. Robbes, P. Bride, "Quelle violence ? Quelle école ?», Cahiers pédagogiques, n 488, Paris, CRAP-

Cahiers pédagogiques, mars-avril 2011, p. 10 